\title{
TITLE:
}

\section{A computational and theoretical analysis of falling frequency VLF emissions}

AUTHOR(S):

Nunn, David; Omura, Yoshiharu

\section{CITATION:}

Nunn, David ...[et al]. A computational and theoretical analysis of falling frequency VLF emissions. Journal of Geophysical Research: Space Physics 2012, 117(A8): A08228.

ISSUE DATE:

2012-08

URL:

http://hdl.handle.net/2433/193711

RIGHT:

(C)2012. American Geophysical Union. 


\title{
A computational and theoretical analysis of falling frequency VLF emissions
}

\author{
David Nunn ${ }^{1,2}$ and Yoshiharu Omura ${ }^{1}$ \\ Received 25 January 2012; revised 12 July 2012; accepted 12 July 2012; published 28 August 2012.
}

[1] Recently much progress has been made in the simulation and theoretical understanding of rising frequency triggered emissions and rising chorus. Both PIC and Vlasov VHS codes produce risers in the region downstream from the equator toward which the VLF waves are traveling. The VHS code only produces fallers or downward hooks with difficulty due to the coherent nature of wave particle interaction across the equator. With the VHS code we now confine the interaction region to be the region upstream from the equator, where inhomogeneity factor $S$ is positive. This suppresses correlated wave particle interaction effects across the equator and the tendency of the code to trigger risers, and permits the formation of a proper falling tone generation region. The VHS code now easily and reproducibly triggers falling tones. The evolution of resonant particle current $J_{E}$ in space and time shows a generation point at $-5224 \mathrm{~km}$ and the wavefield undergoes amplification of some $25 \mathrm{~dB}$ in traversing the nonlinear generation region. The current component parallel to wave magnetic field $\left(J_{B}\right)$ is positive, whereas it is negative for risers. The resonant particle trap shows an enhanced distribution function or 'hill', whereas risers have a 'hole'. According to recent theory (Omura et al., 2008, 2009) sweeping frequency is due primarily to the advective term. The nonlinear frequency shift term is now negative $(\sim-12 \mathrm{~Hz})$ and the sweep rate of $-800 \mathrm{~Hz} / \mathrm{s}$ is approximately nonlinear frequency shift divided by $T_{N}$, the transition time, of the order of a trapping time.

Citation: Nunn, D., and Y. Omura (2012), A computational and theoretical analysis of falling frequency VLF emissions, J. Geophys. Res., 117, A08228, doi:10.1029/2012JA017557.

\section{Introduction}

[2] The problem of the theoretical explanation of the plasma physical mechanisms behind triggered VLF emissions and VLF chorus is one of the most fascinating and difficult in the field and has engaged scientists for many years. There is reasonable agreement on the fundamentals, namely that the process involves nonlinear resonant trapping of cyclotron resonant electrons with energies $\sim \mathrm{keV}$, the power input and free energy for the instability coming from the anisotropy of the hot electron distribution function, normally modeled as a bi-Maxwellian distribution or loss cone type, or a combination of the two.

[3] Early work [Nunn, 1974; Omura and Matsumoto, 1982, 1985; Omura et al., 1991] identified nonlinear resonant electron trapping in the VLF wavefield in an inhomogeneous medium as being the fundamental mechanism. This inhomogeneity derives from the ambient magnetic field as well as from the sweeping frequency of the emissions themselves. In an inhomogeneous medium phase trapped or

${ }^{1}$ RISH, Kyoto University, Kyoto, Japan.

${ }^{2}$ ECS School, Southampton University, Southampton, UK.

Corresponding author: D. Nunn, ECS School, Southampton University, Southampton, SO17 1BJ, UK. (dn@ecs.soton.ac.uk)

(C)2012. American Geophysical Union. All Rights Reserved. 0148-0227/12/2012JA017557 phase locked electrons must keep pace with the changing resonance velocity and thus undergo relatively large changes in energy and magnetic moment. Applying Liouville's theorem the consequence of this is that for trapping times of the order of or greater than a trapping period, a hole or hill appears in the distribution function at the position of the resonant particle trap [Omura et al., 2009; Nunn, 1974]. This results in a large current $J_{E}$ parallel to the wavefield electric field vector giving nonlinear wave growth, normally a multiple of the linear growth rate (when $J_{E}<0$ ). At the same time a large nonlinear current $J_{B}$ parallel to the wave magnetic field appears that is instrumental in setting up the generation region of the emissions and determining the frequency sweep rate [Omura and Nunn, 2011].

[4] It is clear that the plasma physical situation is actually very complex and a full numerical simulation the only answer to the problem. The first successful simulations of the process were in Omura and Matsumoto [1982, 1985] using a 1 dimensional PIC code assuming parallel propagation, and gave a lot of understanding of the processes involved. Earlier Nunn [1974] simulated triggered VLF emissions but using a model for nonlinear resonant particle current employing a strong trapping assumption.

[5] Following this a 1D Vlasov Hybrid Simulation code (VHS) was developed [Nunn, 1990, 1993; Nunn et al., 2005] that was band limited to a bandwidth $\sim 80 \mathrm{~Hz}$ wide which is of order 2-3 trapping widths. This code successfully 
simulated observed triggered VLF emissions and also VLF chorus [Nunn et al., 2009]. Although quite quick to run this code utilized artificial saturation and also matched filtering to handle the substantial frequency gradients across the generation region, though recently this code has run without these features.

[6] In the last few years great progress has been made using numerically intensive broadband 1D PIC codes [Omura et al., 2008, 2009, Hikishima et al., 2009, 2010a]. The first two papers represent simulation of VLF chorus, including retriggering of successive elements, using a full PIC code. The latter paper represents the first simulation of triggered VLF emissions using a full PIC code, which also led to the discovery that nonlinear trapping at high pitch angles gave damping that provided a much needed saturation mechanism.

[7] The Backward Wave Oscillator (BWO) theoretical model of Trakhtengerts [1995, 1999] represents a very different approach. He stated that VLF hiss bands of finite width could at their upper frequency edge develop a step in parallel velocity due to differential particle diffusion rates. This could give greatly enhanced growth rates at wave frequencies whose resonance velocity coincided with that of such a step. However, continuing large growth rates would require the step to move rapidly in velocity space to keep pace with the emission sweeping frequency.

[8] While the basic non relativistic trapping equations and field equations for advancing the wavefield due to the resonant particle current have been known for many years, recently considerable progress has been made in our understanding of the mechanisms behind chorus and VLF emissions. It is not enough to merely simulate these processes. Omura et al. [2007] have extended the theory to the relativistic case and uncovered the phenomenon of turning point acceleration of relevance to the heating problem, whereby the cyclotron resonance velocity can change sign at a high Lorentz factor $\gamma$. Theoretical analyses have shown that the frequency sweep in a chorus element or triggered VLF emission is due primarily to the spatial gradient of frequency/wave number set up in the triggering phase [Katoh and Omura, 2006, 2007; Omura et al., 2008, 2009]. These papers also consider in more detail the stable configuration and structure of the generation region, concluding that the generation point for a riser is at the equator, where for maximum nonlinear power input the inhomogeneity factor should be approximately $S \sim-0.4$ [Omura et al., 2008, 2009; Hikishima et al., 2010a]. Such considerations led to the 'chorus equations' as formulated in the aforementioned papers, enabling them to model effectively the chorus generation region.

[9] The VHS simulations of Nunn showed a strong tendency to trigger rising frequency tones and pointed to the structure for the rising frequency generation region (GR). It would appear that the riser GR is located mainly downstream from the equator (on the side of a hypothetical VLF receiver located at the foot of the field line in question) in the region where both nonlinear currents $J_{E}$ and $J_{B}$ are negative, and the net inhomogeneity factor $S$ is negative, as defined in all the previous literature [Nunn, 1974; Omura et al., 2008, 2009]. The VHS code can produce fallers but only with careful choice of parameters and faller production was less stable and repeatable than for risers [Nunn et al., 2005].
Usually high linear growth rates resulted in a faller by driving the wave profile upstream. Note that upstream is defined in the sense that group velocity is directed from upstream to downstream. The resulting faller GR was found to reside on the upstream side of the equator in the positive inhomogeneity region $S>0$ where $J_{E}<0$ and $J_{B}>0$. These fallers simulated by the VHS code were far from satisfactory. In the case of chorus simulations only downward hooks could be obtained and the resulting sweep rates of simulated fallers were low, about one half of what one would expect for good agreement with observations.

[10] The numerically intensive broadband PIC simulations of [Hikishima et al., 2010a] were found to only trigger risers. Again it was found that the riser GR was located downstream from the equator, this result being in good agreement with CLUSTER observations [Santolik and Gurnett, 2003].

[11] In this paper we shall use the band limited 1D Vlasov VHS code to numerically simulate the triggering of falling tone VLF emissions. To facilitate this, the simulation box and generation region will be confined to the upstream region, ending just $402 \mathrm{~km}$ downstream from the equator. The reason for this approach is threefold. First those fallers that are produced by the VHS code have their generation region in the upstream region. Second both VHS and PIC 1D codes assume unrealistically that wave energy generated upstream by a faller generation region will propagate across the equator without loss into the downstream region, and that the wave particle interaction process there will be phase coherent with that in the upstream region. Confining the simulation zone to the upstream region will suppress the tendency to always trigger risers and eliminate wave phase correlation across the equator and allow stable and reproducible fallers to be simulated, enabling a more detailed look at faller generation region structure and detailed comparison with the wave equations [Katoh and Omura, 2006, 2007; Omura et al., 2008, 2009].

\section{The Vlasov 1D VHS Code}

[12] The code has been described in detail in Nunn [1990, 1993] and the reader is referred to these papers. The code has one spatial dimension $(h)$ assuming parallel propagation or ducting and includes displacement current. The independent variable $h$ is the curvilinear distance along the magnetic field line from the equator or more precisely from the point where $B_{0}(h)$ is a minimum. The direction of wave travel is toward positive $h$ and cyclotron resonant electrons travel toward negative $h$. This variable ' $h$ ' is identical to ' $z$ ' as used in the papers of Nunn and coworkers. The ambient magnetic field $B_{o}(h)$ is a parabolic function of $h$, as is cold plasma density, and the overall inhomogeneity factor $S(h, t)$ includes the term in $\mathrm{d} \omega / \mathrm{d} t$.

[13] The code is narrow band or rather band limited to a frequency range of several trapping frequencies $\sim 80 \mathrm{~Hz}$ about the current mean frequency, and various narrow band assumptions are made throughout. The narrow band field equations in complex vector form are integrated continuously. As soon as a significant overall frequency shift $\sim 3 \mathrm{~Hz}$ is detected in the complex amplitude field, the base frequency is redefined, to ensure that the complex amplitude and current fields remain slowly varying. 
[14] The resonant particle current field is noisy and generates broadband noise in the simulation. To keep the simulation band limited the wave and current fields are band pass filtered by DFT/IDFT. Out of band noise is not properly handled by the code grid system and not provided with resonant particles. It was proved rigorously in Katoh and Omura [2006, 2007] that frequency sweep rate is due primarily to established frequency gradient across the interaction region, and this has been confirmed by the simulation [Omura et al., 2008, 2009; Nunn et al., 2005, 2009]. This creates a problem with band pass filtering since too narrow a filter will clip the wavefield at the ends of the simulation box. This is overcome by using a matched filter in which the mean linear frequency gradient is divided out, the band pass filter applied and then the frequency gradient term multiplied back in. Recently the code has been run without this feature.

[15] It was found that the narrow band simulation achieved very high amplitudes on occasions and the code is often run with artificial saturation imposed in order to control wave amplitudes. In this work the simulation will be allowed to saturate naturally.

[16] The simulation box in the spatial dimension $h$ about the equator is set up to include the lesser of the trapping region at the maximum wave amplitude or the region where resonant particle fluxes/linear growth rate are significant. In the original code the perpendicular velocity dimension was deemed relatively unimportant and this dimension was treated coarsely ( 3 grid points) on the grounds that most of the nonlinear effects derive from a relatively narrow range of pitch angles 40-60 degrees. It should be noted though that in recent work [Hikishima et al., 2010a] a full range of perpendicular velocity, properly resolved, was found to be necessary to model saturation and sideband instability properly, and so in this application we will employ a wide range of pitch angles.

[17] The parallel velocity grid in $V_{\|}$is always centered on the local resonant velocity $V_{R}(h, t)$ and has a width corresponding to the simulation bandwidth plus several trapping widths at the maximum wave amplitude. Since wave frequency is a marked function of space and time the velocity grid is in constant motion.

[18] The VHS technique itself is concerned with how the resonant particle current is calculated. Note that protons may be assumed immobile and the linear cold plasma electrons are treated analytically through the field equation. At any one time only resonant or near resonant electrons are being considered in the code. The simulation box is filled with simulation particles (SP's) at a density which should be greater than approximately 1.2 particles per hypercube. The SP's represent test particles embedded in the Vlasov fluid. They are followed forwards continuously in time throughout the simulation. Trajectories are not restarted at grid points but only end at the end of the run or when that particle leaves the phase space simulation box. By Liouville's theorem $F$ is conserved along these phase space trajectories and is thus known. At each time step then, using a simple linear interpolator, the velocity distribution function $F$ is interpolated from particles onto the phase space grid, whence resonant particle current is readily calculated.

[19] One may be concerned that many grid points will not have a simulation particle in adjacent cells. However, by Liouville's theorem density of simulation particles itself is also conserved and voids happen quite rarely, and are easily fixed by interpolation from the next grid points. Due to the inhomogeneous nature of the chorus/VLF emission problem, particles are constantly being swept into resonance, i.e., Vlasov fluid flows into the box. When this occurs new simulation particles need to be inserted into the phase fluid at an appropriate density.

\section{The Faller Simulation}

[20] In presenting the results we shall employ dimensionless distance $\tilde{h}=h /\left(c \Omega_{e 0}^{-1}\right)$, dimensionless time $\tilde{t}=t \Omega_{e 0}$, dimensionless frequency $\tilde{\omega}=\omega / \Omega_{e 0}$, and dimensionless wave amplitude $\tilde{B}_{w}=B_{W} / B_{o e q}$. The simulation is for $\mathrm{L}=4.2$, and a cold plasma electron density of $N_{e}=100 / \mathrm{cc}$, and so inside the plasmapause. The corresponding equatorial electron plasma frequency is then $89807 \mathrm{~Hz}$, the equatorial electron gyrofrequency $11878 \mathrm{~Hz}$, and initial triggering frequency $4929 \mathrm{~Hz}$.

[21] The simulation box was set up to cover a spatial range from $\tilde{h}=-2500$ to $+100(-10047 \mathrm{~km}$ to $+402 \mathrm{~km})$ relative to the equator, which more than covers the trapping region upstream. Even a moderate extension into the negative inhomogeneity region allowed risers to develop. The number of spatial grid points was 2048 allowing ample resolution of the time and spatial scales involved related to trapping frequency and bandwidth. The number of grid points in gyrophase is 32 and in parallel velocity 80 . Ten grid points in perpendicular velocity are used covering the range 2075 degrees of pitch angle. The trapping frequency at the highest pitch angle is $\tilde{\omega}_{t r}=7.5 \times 10^{-3}(89 \mathrm{~Hz})$. The global bandpass filter has a width $\tilde{\omega}_{B}=0.087(1040 \mathrm{~Hz})$ and the matched filter a width of $\tilde{\omega}_{B M}=0.0089(106 \mathrm{~Hz})$. The zero order distribution function consisted of four superimposed bi-Maxwellians spaced in energy. Of these the dominant component has a perpendicular thermal energy of $5 \mathrm{keV}$ and a parallel thermal energy of $1.5 \mathrm{keV}$ giving a ratio of perpendicular to parallel temperature of $\sim 3.3$. The zero order distribution function was normalized to give a linear growth rate of $160 \mathrm{~dB} / \mathrm{s}$ at the start frequency at the equator, which is rather higher than usually used to trigger risers in simulations $(\sim 90 \mathrm{~dB} / \mathrm{s})$ due in part to the restricted size of the interaction region, but is however consistent with results in Nunn et al. [2005] where high growth rates gave fallers, and is also consistent with high values of path integrated linear growth rates up to $800 \mathrm{~dB}$ calculated from THEMIS electron distribution function observations [Li et al., 2010]. To some extent the required linear growth rate is dictated by the code itself. A certain minimum linear growth rate is required in order for a self-sustaining generation region to be possible and the value used in the code must be set such that triggered emissions result. This degree of linear instability gives a total hot electron density corresponding to the dominant bi Maxwellian of $N_{h}=7.4210^{4} / \mathrm{m}^{3}$, or a ratio of $N_{h} / N_{e}=$ 0.00074 .

[22] The code requires a triggering signal. It can be triggered from random noise but here we use a $\mathrm{CW}$ pulse, duration $\tilde{t}=1.34 \times 10^{5}(340 \mathrm{~ms})$, frequency $\tilde{\omega}=0.415$ $(4929 \mathrm{~Hz})$ and amplitude $\tilde{B}_{w}=1.15 \times 10^{-5}(5 \mathrm{pT})$.

[23] For reasons of space we shall only analyze one simulated faller, though these faller simulations were very stable and reproducible for a wide range of input parameters. 


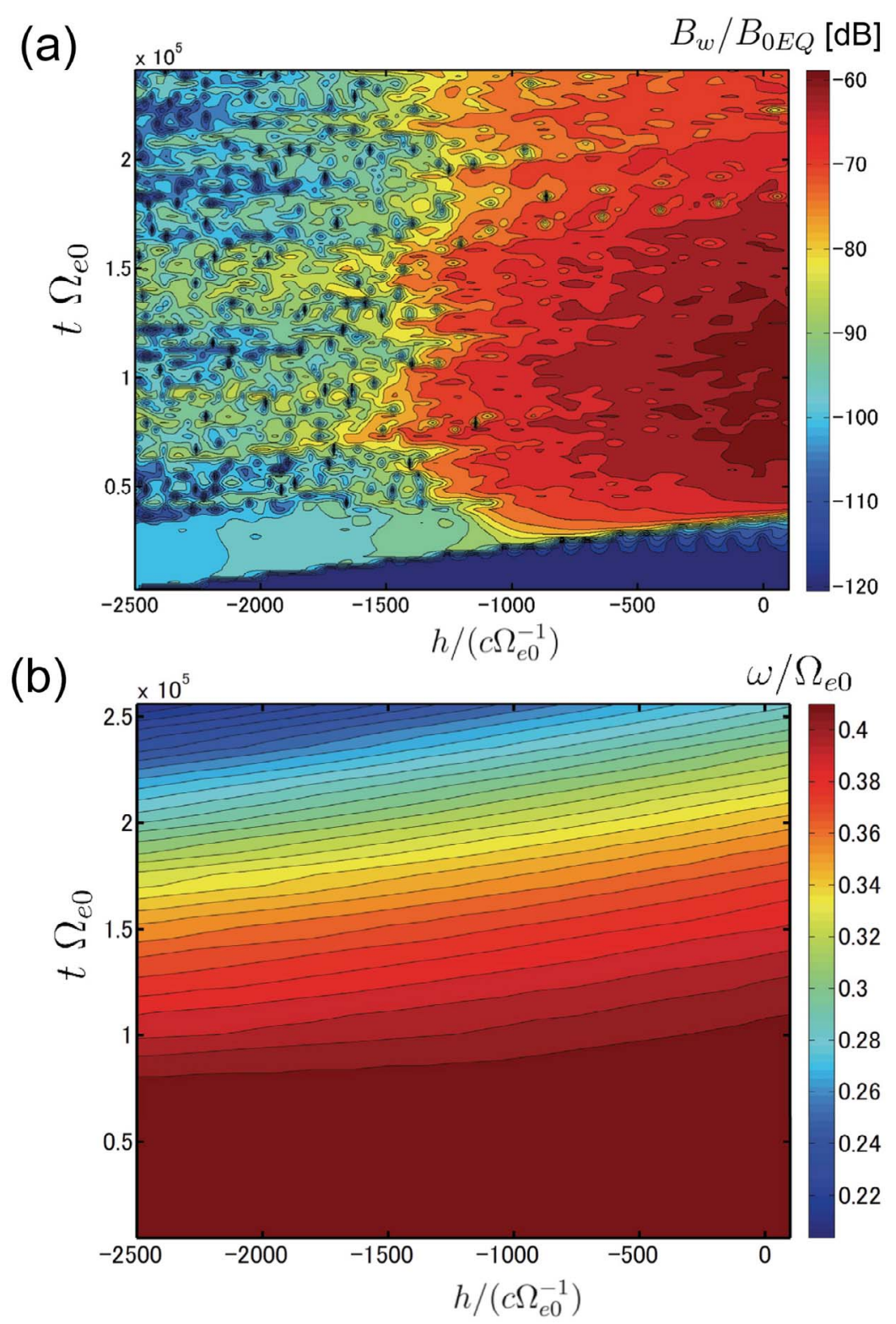

Figure 1. (a) Amplitude history of the entire faller simulation, presented as a contour plot of normalized wave amplitude in $\mathrm{dB}$ in the dimensionless $\tilde{h}-\tilde{t}$ plane. (b) History of smoothed localized normalized frequency $\left(\tilde{\omega}=\omega / \Omega_{e 0}\right)$ in the same plane, where it is seen that a substantial positive spatial frequency gradient is established which is the cause of the falling frequency.

Figure 1a presents a history of normalized wave amplitude $20 \log _{10}\left(B_{w} / B_{o}\right)$ in $\mathrm{dB}$ for the whole simulation, plotted in the normalized $\tilde{z}-\tilde{t}$ plane. The weak incoming signal is clearly apparent. At $\tilde{t}=0.5 \times 10^{5}(0.67 \mathrm{~s})$ a generation region for a faller is established, which remains as a quasi-static nonlinear entity. After $\tilde{t}=1.5 \times 10^{5}(2.01 \mathrm{~s})$, the whole GR decays due to the decreasing particle flux, since resonance velocity increases with falling frequency. Figure $1 \mathrm{~b}$ is a similar plot showing the history of smoothed local frequency for the entire simulation. From about $\tilde{t}=0.8 \times 10^{5}(1.07 \mathrm{~s})$ a pronounced positive spatial gradient of frequency becomes established across the GR and it is this that produces the temporal negative frequency sweep rate observed at a fixed location.
[24] Figure $2 \mathrm{~b}$ is a frequency time spectrogram of the wave data stream emerging from the right hand end of the simulation box at $\tilde{h}=+100(402 \mathrm{~km})$. The diagram is produced from overlapping Hamming weighted 350 point DFT's. The quantity plotted is DFT spectral power in dB's relative to an arbitrary level. The plot is 'flood filled' to render the emission itself clearly visible. The emission is clearly stable and achieves a sweep rate of $\partial \tilde{\omega} / \partial \tilde{t}=8.3 \times$ $10^{-7}(\sim-800 \mathrm{~Hz} / \mathrm{s})$. It will be noted that there is a pulsation or sideband structure related to sideband instability. According to theoretical analyses in Nunn [1986] in a positive inhomogeneity one expects lower sideband instability/ upper sideband damping with optimum separation of the order of the trapping frequency. A rather different view of 
(a)

$h /\left(c \Omega_{e 0}^{-1}\right)=-1300$

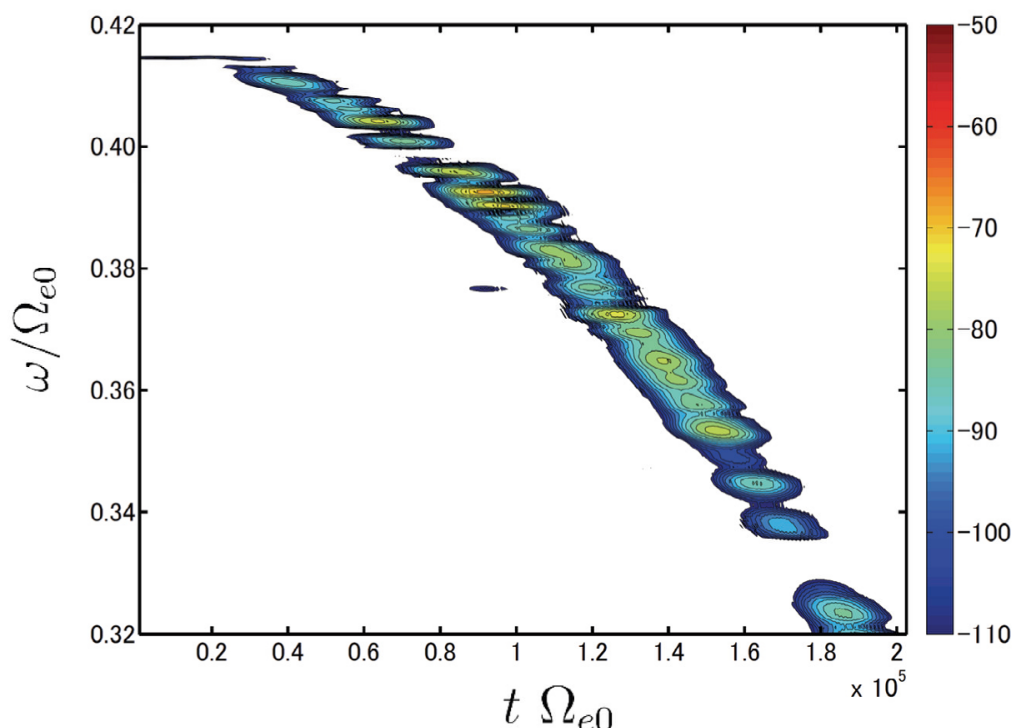

(b) $h /\left(c \Omega_{e 0}^{-1}\right)=100$

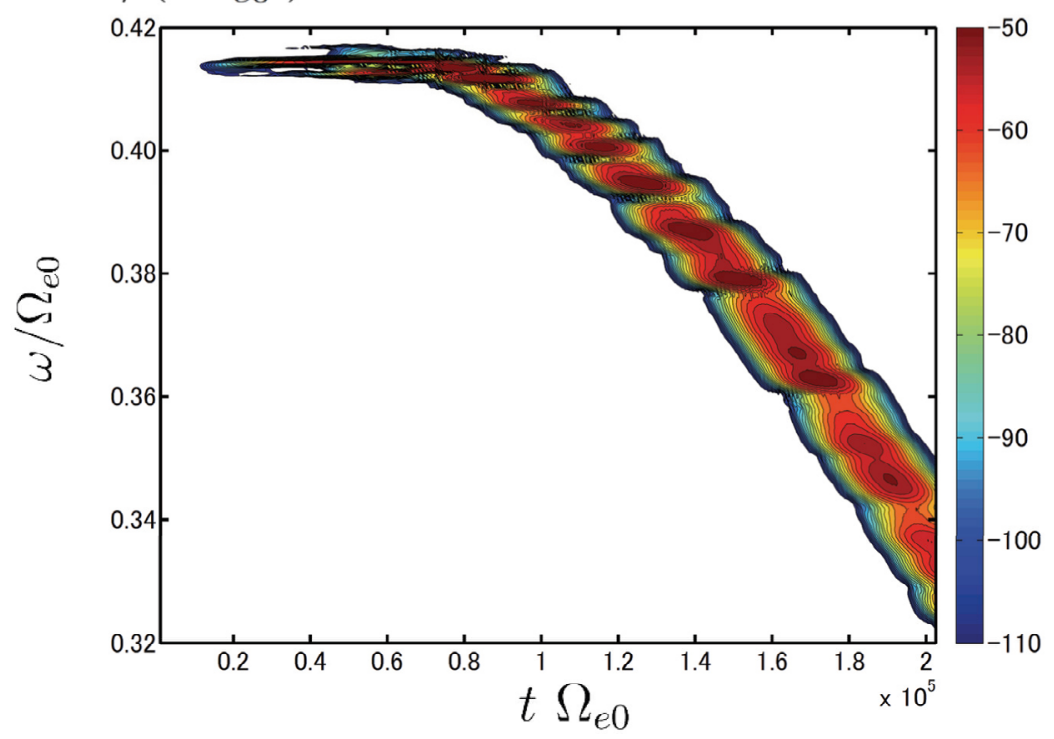

Figure 2. (a) Spectrogram of wavefield at the generation point $\tilde{h}=-1300$. The plot is of DFT spectral power in dBs relative to an arbitrary level, in the normalized frequency $\tilde{\omega}, \tilde{t}$ plane. The plot is flood filled for presentation purposes. The initial triggering pulse and emission sideband structuring are clearly visible. The sweep rate is $\sim-800 \mathrm{~Hz} / \mathrm{s}$. (b) A similar spectrogram at the simulation box exit $\tilde{h}=+100$. The difference in power between these two represents the nonlinear amplification $25 \sim 30 \mathrm{~dB}$ in traversing the generation region.

the mechanism for falling frequency is to regard it as being due to the progressive transfer of spectral power to lower sidebands. However, the assertion that the falling frequency is due to an established positive gradient of frequency is well established [Katoh and Omura, 2006, 2007], and it is not clear that any additional mechanism is required. Having said that the lower sideband instability must be an important part of the overall plasma physical process and this matter requires further investigation.
[25] Using the same color scale and $\tilde{h}-\tilde{t}$ box, Figure 2a plots the emission history at the point $\tilde{h}=-1300$ $(-5224 \mathrm{~km})$ which is approximately the generation point or upstream edge of the GR. The initial pulse and negative frequency offset are apparent as well as sideband structure and overall emission shape in the frequency time plane. Overall wave amplitudes at the generation point are roughly $25 \mathrm{~dB}$ lower than at the downstream exit point, this difference of course representing the nonlinear amplification undergone in traversing the GR. 


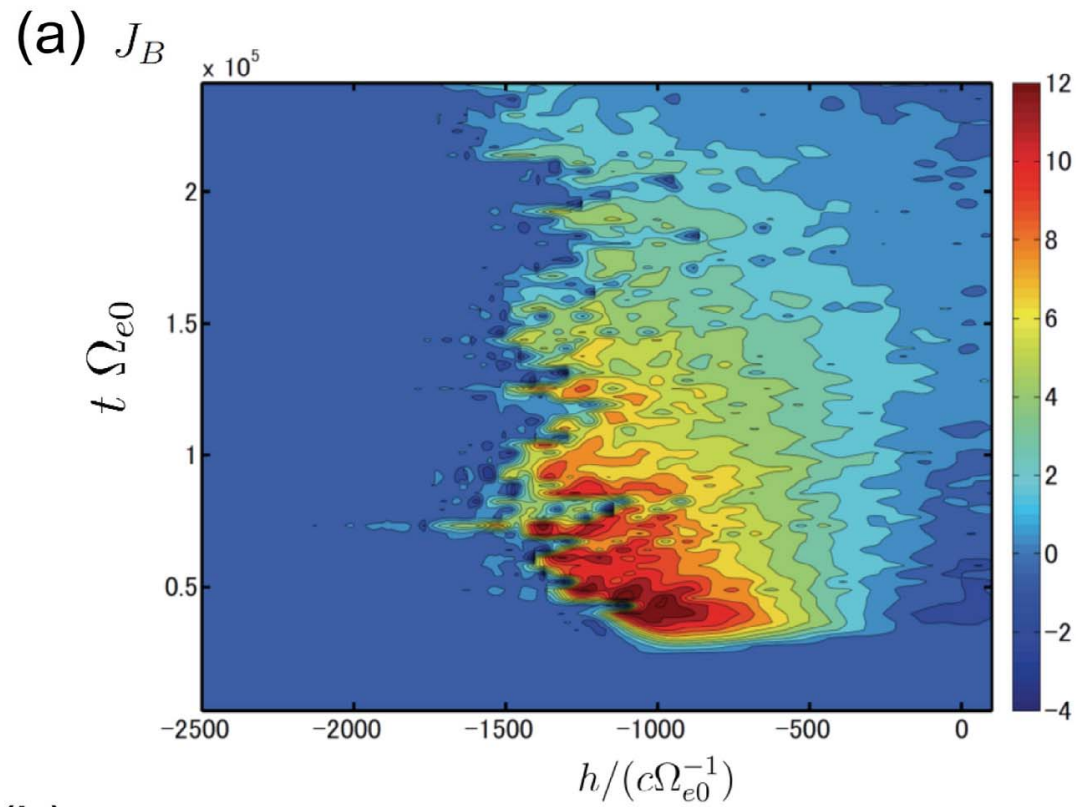

(b) $-J_{E}$

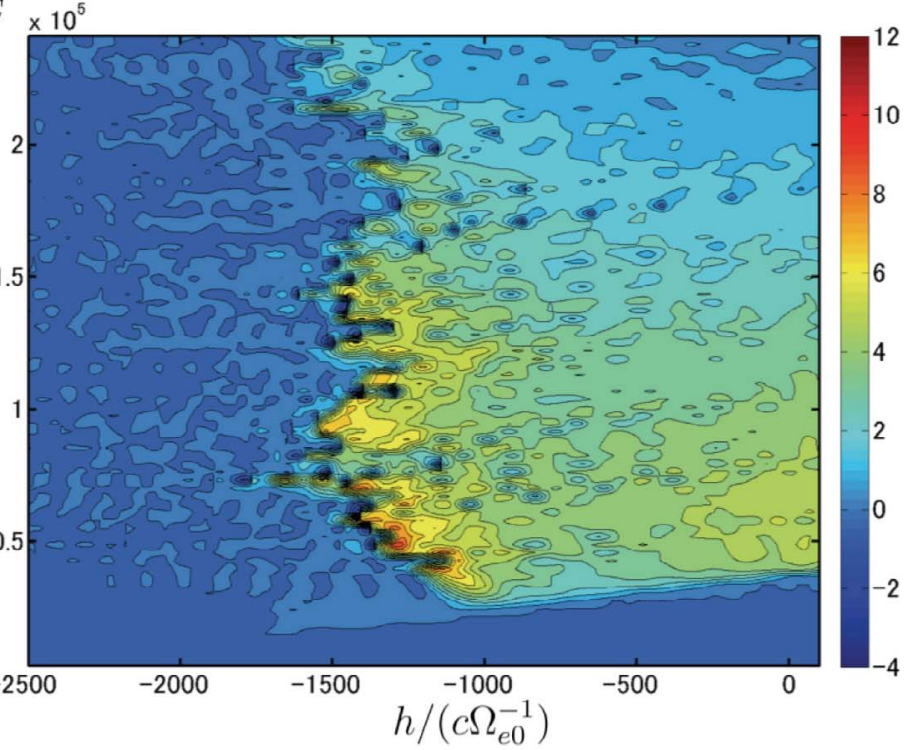

Figure 3. (a) History in the $\tilde{h}-\tilde{t}$ plane of the component of resonant particle current parallel to wave magnetic field, $J_{B}$. Unity current corresponds to the equatorial current for a field of $24 \mathrm{pT}$ for linear interaction. For a faller $J_{B}$ is now positive in contrast to the case of a riser where it is negative. Large values $\sim+10$ occur at $\tilde{h} \sim-1000$ in the triggering phase. Current $J_{B}$ shifts wave phase and causes the spatial gradient of frequency/wave number to be set up during the triggering phase. (b) History of $J_{E}$ the current component parallel to wave electric field. This component is responsible for nonlinear growth. Maximum growth is concentrated in the region of $\tilde{h} \sim-1300$ which is the leading edge of the generation region and we identify this as the generation point.

[26] Figure $3 \mathrm{~b}$ shows a similar history for the nonlinear resonant particle current component anti-parallel to wave electric field, namely $-J_{E}$. This is the quantity responsible for nonlinear wave growth, expressed as a multiple of the linear value of $-J_{E}$ which would be expected at the equator for an amplitude of $24 \mathrm{pT}$. Values $\sim 6$ times this linear figure are achieved. It will be noticed that there is very strong nonlinear growth in the region of $\tilde{h}=-1300(-5224 \mathrm{~km})$, which clearly is the initial generation point of the faller as indicated in the theory in Omura et al. [2009].

[27] Figure 3a shows a similar history of the component of nonlinear resonant particle current $J_{B}$ parallel to wave magnetic field, in the same units as $J_{E}$. As expected in this positive $S$ region $J_{B}>0$, and the highest values are achieved at $\tilde{h} \sim-1000(\sim-4019 \mathrm{~km})$ a little downstream from the generation point. Most recent work [Omura and Nunn, 2011] shows that $J_{B}$ plays a key role in the setting up of 

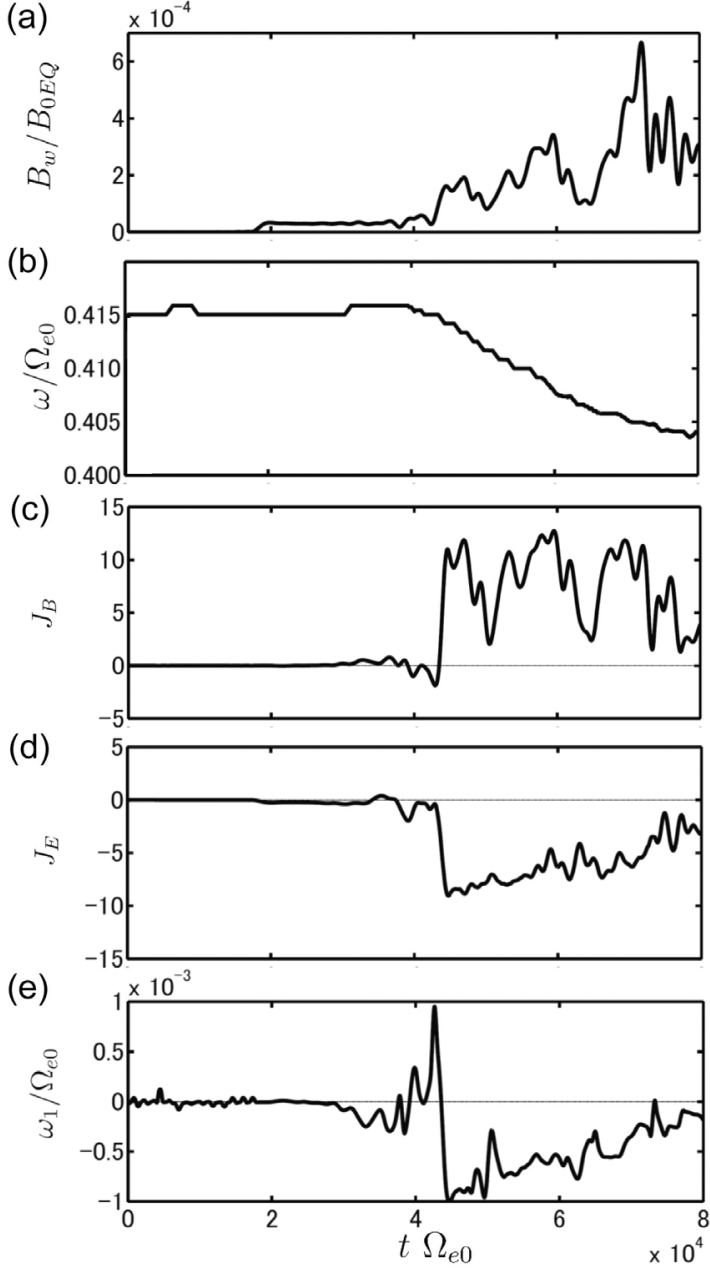

Figure 4. Plots showing key variables as functions of dimensionless time at the generation point $\tilde{h} \sim-1300$ during the triggering phase (a) Normalized wavefield amplitude $B_{\mathrm{w}} / B_{0 e q}$. (b) Normalized frequency $\left(\omega / \Omega_{e 0}\right)$. (c) Resonant particle current $J_{B}$. (d) Resonant particle current $J_{E}$. (e) Normalized nonlinear frequency shift $\left(\omega_{1} / \Omega_{e 0}\right)$. The currents $J_{B}$, $J_{E}, \omega_{1}$, and $B_{\mathrm{w}}$ all rise quite abruptly at the triggering time just past $\tilde{t}=4 \times 10^{4}$. Current $J_{E}$ sustains negative values giving nonlinear growth. Nonlinear frequency shift $\omega_{1}$ achieves a steady value $-10 \mathrm{~Hz}$ over a short time period $\sim$ trapping time setting up the spatial gradient of frequency.

the generation region and in particular the spatial gradient of frequency/wave number, but plays only a small role in the established quasi static generating region. Following the development in the above paper it is of considerable interest to examine conditions at this generating point in the case of a faller and to understand how the crucial gradient of frequency is set up. Figure 4a shows the time development of normalized wave amplitude $B_{w} / B_{o}$ at $\tilde{h}=-1300$ during the initial triggering phase only. It is seen that an initial approximate amplitude of $B_{w} / B_{o} \sim 3 \times 10^{-4}$ is reached, sufficient for nonlinear trapping. At $\tilde{t} \sim 4.2 \times 10^{4}(0.56 \mathrm{~s})$, the triggering time, a sustained negative value of nonlinear current $J_{E}$ abruptly appears giving a substantial nonlinear growth rate (Figure $4 \mathrm{~d}$ ), and almost simultaneously a sustained but more variable positive value of nonlinear current $J_{B}$ (Figure $4 \mathrm{c}$ ) is seen. The units of $J_{B}$ and $J_{E}$ are as defined for Figure 3. Figure $4 \mathrm{~b}$ plots localized smoothed wave frequency observed at $\tilde{h}=-1300$ and clearly there is a steady fall in frequency which starts immediately at the triggering time.

[28] In Nunn [1974] and Omura et al. [1991] it was shown that the time progression of wave additional phase $\varphi$ was given by the equation

$$
\partial \varphi / \partial t+V_{g} \partial \varphi / \partial h=\omega_{1}
$$

where the nonlinear frequency shift term $\omega_{1}$ is given by

$$
\omega_{1}=-\mu_{0} V_{g} J_{B} /\left(2 B_{w}\right)
$$

Figure $4 \mathrm{e}$ shows the time development of the normalized value of nonlinear frequency shift $\omega_{1} / \Omega_{e 0}$ as a function of time $\tilde{t}$ at triggering point $\tilde{h}=-1300$. The initial fall and marked positive peak correspond to quite small wave amplitudes. As soon as significant wave amplitudes appear $\omega_{1} / \Omega_{e 0}$ attains a substantial negative value $\sim-10^{-3}$ $(\sim-11.8 \mathrm{~Hz})$ which is sustained throughout the triggering phase.

[29] It is a useful exercise to inspect the resonant particle distribution function $F_{\text {res }}$ as a function of gyrophase $\zeta$ and $\left(V_{\|}-V_{R}\right) / V_{t r}$, where the trapping width $V_{\mathrm{tr}}=2 \omega_{\mathrm{tr}} / k, \omega_{\mathrm{tr}}$ being defined in section 4 and evaluated at the equator for a wave amplitude of $24 \mathrm{pT}$ and for average perpendicular velocity. It was shown in Omura et al. [2008] that for $|S|=$ 0.4 the whole width of the trap in $V_{\|}$is $V_{\text {tr }}$, and the width of the simulation box in $V_{\|}$in the code is twice this at $2 V_{\text {tr }}$. Figure 5 plots $F_{\text {res }}$ in arbitrary units at a pitch angle of $60^{\circ}$, and at $\tilde{t}=10^{5}(1.34 \mathrm{~s}), \tilde{h}=-664(-2670 \mathrm{~km})$ which is in the middle of the Generation Region where the trapped particles are tightly locked at the phase trapping angle. The trapped particle bunch is clearly apparent and has a phase in the quadrant $\zeta=180 \sim 270$ degrees as expected for positive $S$.

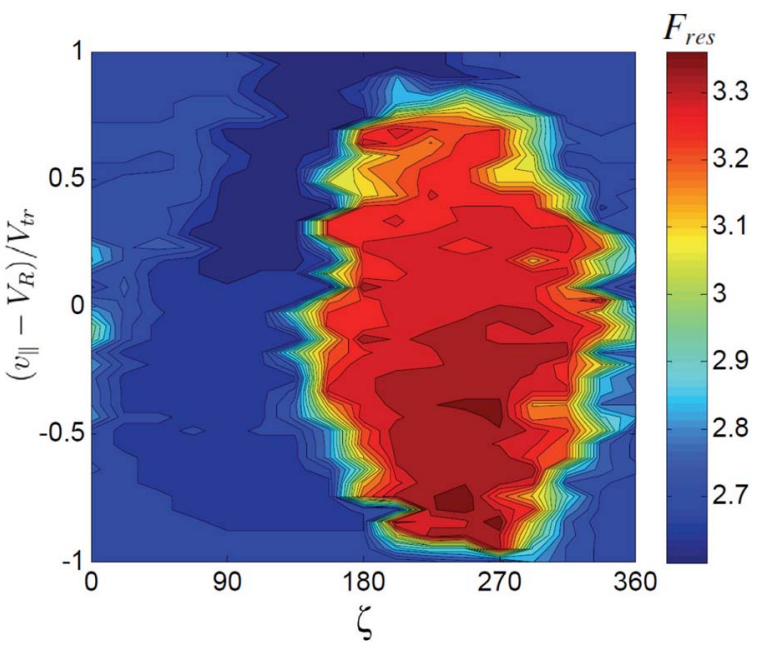

Figure 5. Plot of resonant particle distribution function in the $\zeta-V_{\|}$plane for a pitch angle of 60 degrees. The bunch of stably and strongly trapped electrons will be noted with a mean distribution function $F_{\text {res }}$ some $18 \%$ above that of the unperturbed value $F_{0}$. The surrounding untrapped electrons have distribution function values $\sim F_{0}$. 


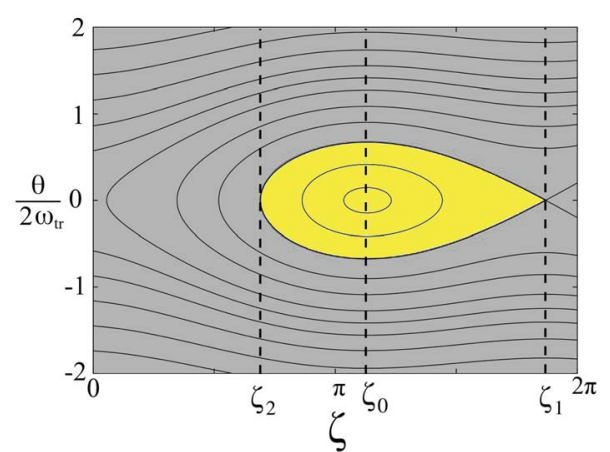

Figure 6. Illustration of resonant particle dynamics in the $\zeta-\theta$ plane. The passing particles sweep through resonance and have a distribution function that is relatively unperturbed. The stably trapped particles have a distribution function very different from the unperturbed value, thus presenting a 'hole' or 'hill' in velocity space.

The trapped particles are characterized by a mean value of $F_{\text {res }}$ some $18 \%$ higher than the ambient $F_{0}$. The surrounding untrapped particles have a relatively unperturbed distribution function $\sim F_{0}$. We thus have an 'electron hill' corresponding to a $Q$ factor in the nonlinear current model [Omura et al., 2009] of -0.2 . This is in contrast to the riser case where in a negative inhomogeneity an electron hole is found.

[30] Since in this simulation we have a wide range of pitch angles, a useful definition is the quantity $\mathrm{G}_{\mathrm{I}}$ as a function of $\zeta$ and $V_{\|}$

$$
G_{I}\left(\zeta, V_{\|}\right)=\int V_{\perp}^{2} F_{r e s} d V_{\perp}
$$

where $\zeta$ is gyrophase relative to $B_{w}$, and $G_{I}$ has the significance that

$$
J_{B}-i J_{E}=-e \iint \exp (i \zeta) G_{I} d \zeta d V_{\|}
$$

Here $G_{I}$ represents a pitch angle average of resonant particle distribution function and will also exhibit here an elevation or 'hill' inside the resonant particle trap of some $7 \%$. This occurrence of a distribution function hill is readily understood. In a positive inhomogeneity trapped particles decrease in energy and magnetic moment [Nunn, 1974]. In an unstable anisotropic bi Maxwellian zero order distribution function application of Liouville's theorem confirms that trapped particles have a higher distribution function than surrounding untrapped particles, whose distribution function is close to $F_{o}$.

\section{Theoretical Analysis of the Falling Tone Emission}

[31] Chorus generation is clearly a nonlinear process and the underlying nonlinear dynamics derives from nonlinear trapping of cyclotron resonant electrons in the inhomogeneous medium. The theory of trapping in inhomogeneous media was developed in Nunn [1974, 1990] and Omura et al.
[2008, 2009]. Following the development in Omura et al. [2009], we may express the equation of motion for $\zeta$ as

$$
\frac{d^{2} \zeta}{d t^{2}}=\omega_{t r}^{2}(\sin \zeta+S)
$$

where $\omega_{\text {tr }}$ is the trapping frequency given by

$$
\omega_{t r}=\chi \sqrt{k V_{\perp 0} \Omega_{w} / \gamma}
$$

and where $V_{\perp 0}$ is the average perpendicular velocity. The parameter $\Omega_{\mathrm{w}}=\mathrm{e} B_{w} / \mathrm{m}_{0}$ is the normalized wave amplitude, where $-\mathrm{e}$ and $\mathrm{m}_{0}$ are the charge and rest mass of an electron. The quantity $\chi$ is given by

$$
\chi^{2}=\left(1+\xi^{2}\right)^{-1} ; \xi^{2}=\omega\left(\Omega_{e}-\omega\right) / \omega_{p e}^{2}
$$

and $\gamma$ is the usual relativistic Lorentz factor. The parameter $S$ is the inhomogeneity factor [Nunn, 1974; Omura et al., 2008, 2009] given by

$$
S=-\frac{1}{s_{0} \omega \Omega_{w}}\left(s_{1} \frac{\partial \omega}{\partial t}+c s_{2} \frac{\partial \Omega_{e}}{\partial h}\right)
$$

where

$$
\begin{gathered}
s_{0}=\frac{\chi V_{\perp o}}{\xi c} \\
s_{1}=\gamma\left(1-V_{R} / V_{g}\right)^{2}
\end{gathered}
$$

and

$$
s_{2}=\frac{1}{2 \xi \chi}\left\{\frac{\gamma \omega}{\Omega_{e}}\left(\frac{V_{\perp o}}{c}\right)^{2}-\left[2+\Lambda \frac{\chi^{2}\left(\Omega_{e}-\gamma \omega\right)}{\Omega_{e}-\omega}\right] \frac{V_{R} V_{p}}{c^{2}}\right\}
$$

In the above equations $V_{p}$ is the phase velocity given by

$$
V_{p}=\omega / k=c \chi \xi
$$

and $V_{R}$ is the cyclotron resonance velocity which is the root of the equation [Omura and Nunn, 2011]

$$
\omega-k V_{\|}=\Omega_{e} / \gamma
$$

We have incorporated the variation of the cold electron density $N_{e}(h)$ along the magnetic field line as $N_{e}(h)=$ $N_{e 0} \Omega_{\mathrm{e}}(h) / \Omega_{\mathrm{e} 0}$ where $N_{e 0}$ and $\Omega_{\mathrm{e} 0}$ are respectively the cold electron density and the electron gyrofrequency at the equator. It may be shown that $\Lambda=\omega / \Omega_{e}$ for this inhomogeneous electron density model, while $\Lambda=1$ for the constant electron density model as assumed by Omura et al. [2009].

[32] To maintain a falling tone, clearly from (8) at the equator

$$
S=-\frac{1}{s_{o} \omega \Omega_{w}} s_{1} \frac{\partial \omega}{\partial t}
$$

where we need $S>0$. Indeed in the present simulation where $h<0, S>0$ everywhere. The second order resonance condition for stably trapped electrons is given from (5) as

$$
\sin \zeta_{0}+S=0
$$


where $\zeta_{0}$ is the phase trapping or phase locking angle. For $S>0$ we have $\sin \zeta_{0}<0$ and the phase trapping angle will lie in the quadrant $\pi<\zeta_{0}<3 \pi / 2$. Resonant electron trapping dynamics is well known and has been extensively discussed in the literature [Nunn, 1974]. This is illustrated in Figure 6 which illustrates particle phase trajectories in the gyrophase $\zeta-\theta$ plane, where $\theta=k\left(V_{\|}-V_{R}\right)$, assuming the wavefield is narrow band and that inhomogeneity remains constant. The trap is centered at the resonance velocity and at the phase locking angle $\zeta_{0}$. The so-called 'passing' or untrapped electrons sweep through resonance as a result of the inhomogeneity. Electrons which have been trapped for multiple trapping periods will undergo relatively large changes in energy and magnetic moment and will have a distribution function substantially different from surrounding untrapped electrons. It may be readily shown that for $S>0$ and for linearly unstable zero order distribution functions this will give $\mathrm{d} F>0$ or a 'hill' in distribution function located at the particle trap in velocity space. The fractional lowering of distribution function averaged over the trap in velocity space is represented by the quantity $Q$ [Omura et al., 2009]. The distribution function outside the trap will be relatively close to the unperturbed value. From Liouville's theorem we may write an expression for $Q$ which is the trap average of

$$
Q=A v\left\{\left[F_{0}(\mu, W)-F_{0}(\mu-\Delta \mu, W-\Delta W)\right] / F_{0}(\mu, W)\right\}
$$

where $W$ is electron energy, $\mu$ is electron magnetic moment and $F_{0}$ is the unperturbed distribution function. The quantities $\Delta W$ and $\Delta \mu$ are the integrated past changes in energy and magnetic moment of an electron located at that position in configuration space. This quantity $Q$ can only be properly determined from numerical simulation. Note that positive $Q$ corresponds to a distribution function depression or hole. In the case of a faller we have a distribution function hill and $Q<0$ and for a riser a hole and $Q>0$.

[33] The relatively simple nature of nonlinear trapping dynamics when $S$ is slowly varying opens the way to constructing simple models of nonlinear current [Omura et al., 2008, 2009; Nunn, 1974]. Assuming that the depression or elevation of distribution function in the resonant particle trap is the dominant contributor to current in the integration over velocity space, we may write the approximate relation

$$
J_{B}-i J_{E}=+e \int_{V_{\perp}} Q V_{\perp}^{2} \int_{0}^{2 \pi} \int_{-\infty}^{\infty} F\left(V_{\|}, \zeta, V_{\perp}\right) \exp (i \zeta) d V_{\|} d \zeta d V_{\perp}
$$

where the quantity $F$ has the definition $F=0$ outside the trap boundary in velocity space and $F=F_{0}$, the unperturbed distribution function, inside the trap. In performing the above integral then we take the $V_{\perp}$ dependence of $F$ to be a delta function $\delta\left(V_{\perp}-V_{\perp 0}\right)$ located at $V_{\perp 0}$. We expect trapping to be most significant at intermediate pitch angles since $\mathrm{S}$ becomes large at low and high values of perpendicular velocity [Nunn, 1974]. It is thus a reasonable approximation to consider wave particle effects to derive from a narrow range of pitch angles. We may now write [Omura et al., 2008, 2009]

$$
J_{B}-i J_{E}=+e Q V_{\perp 0}^{2} \int_{0}^{2 \pi} \int_{-\infty}^{\infty} g_{t}\left(V_{\|}, \zeta\right) \exp (i \zeta) d V_{\|} d \zeta
$$

where inside the trap $g_{\mathrm{t}}\left(V_{\|}, \zeta\right)=G\left(V_{\|}\right)$represents the integral over perpendicular velocity of the ambient distribution function $F_{0}$. Outside the trap $g_{\mathrm{t}}$ is zero. Following Omura et al. [2008, 2009], we perform the integral over the trap to obtain an expression for $J_{E}$

$$
J_{E}=-J_{0} \int_{\zeta_{2}}^{\zeta_{1}}\left[\cos \zeta_{1}-\cos \zeta+S\left(\zeta-\zeta_{1}\right)\right]^{\frac{1}{2}} \sin \zeta d \zeta
$$

where

$$
J_{0}=(2 e)^{3 / 2}\left(m_{0} k \gamma\right)^{-1 / 2} V_{\perp 0}^{5 / 2} \chi Q G B_{W}^{1 / 2}
$$

The phase angles $\zeta_{1}$ and $\zeta_{2}$ define the boundary of the trapping wave potential as described by Omura et al. [2008]. The integral in (19) has a maximum value of 0.975 at $S=0.4$ which represents the optimum inhomogeneity for nonlinear growth. Thus

$$
\left(-J_{E}\right)_{\max }=-0.975(2 e)^{3 / 2}\left(m_{0} k \gamma\right)^{-1 / 2} V_{\perp 0}^{5 / 2} \chi Q G B_{W}^{1 / 2}
$$

At this value of inhomogeneity the corresponding value of $J_{B}$ is $-1.3 J_{0}$. The narrow band in-phase wave equation [Nunn, 1974, Omura et al., 2008] may be written as

$$
d B_{w} / d t=\partial B_{w} / \partial t+V_{g} \partial B_{w} / \partial h=-\left(\mu_{0} V_{g} / 2\right) J_{E}
$$

enabling us to define a localized nonlinear growth rate $\Gamma_{N}$ through

$$
d B_{w} / d t=\Gamma_{N} B_{w}
$$

The quantity $G$ may be conveniently expressed as a Maxwellian function

$$
G=\frac{N_{h}}{2 \pi^{3 / 2} U_{t \|} U_{\perp o}} \exp \left(-\frac{\gamma^{2} V_{R}^{2}}{2 U_{t \|}^{2}}\right)
$$

where $U_{\perp 0}=\gamma V_{\perp 0}, U_{t \|}$ is the relativistic thermal momentum, and we have normalized $G$ to the density of hot electrons $N_{h}$. From (21)-(24) we now obtain the following key expression for nonlinear growth rate $\Gamma_{N}$ in the form

$$
\Gamma_{N}=-\frac{0.975 Q \omega_{p h}^{2}}{2}\left(\frac{\zeta}{\Omega_{w} \omega}\right)^{\frac{1}{2}} \frac{v_{g}}{U_{t \|}}\left(\frac{V_{\perp o} \chi}{c \pi \gamma}\right)^{\frac{3}{2}} \exp \left(-\frac{\gamma^{2} V_{R}^{2}}{2 U_{t \|}^{2}}\right)
$$

where $\omega_{p h}$ is the plasma frequency of the hot electrons given by $\omega_{p h}^{2}=N_{h} \mathrm{e}^{2} /\left(\varepsilon_{0} \mathrm{~m}_{0}\right)$ where $\varepsilon_{0}$ is the permittivity of free space. The reader is reminded that this expression is a maximal value and where inhomogeneity factor $S$ is not equal to +0.4 a reduction factor will apply. The model requires that simple trapping takes place and the field bandwidth does not exceed the trapping frequency. The factor $Q$, the fractional decrease (increase if $Q<0$ ) of the mean distribution function in the trap depends on the entire trapping history of the process and strictly requires a full numerical simulation to determine it.

[34] We now turn our attention to the time development of wave phase. The narrow band wave equation furnishes the expression for additional wave phase $\varphi(h, t)$ in (1). 
Following Katoh and Omura [2006, 2007] defining additional frequency $\omega^{\prime}=\partial \varphi / \partial t$, we obtain

$$
\partial \omega^{\prime} / \partial t+V_{g} \partial \omega^{\prime} / \partial h=\partial \omega_{1} / \partial t
$$

In a quasi-static generation region the right hand term cannot be significant, and hence frequency sweep rate must derive almost entirely from the advective term [Omura et al., 2008, 2009]. The question of how the nonlinear current field $J_{B}(h, t)$ sets up this spatial gradient of wave number during the triggering phase is addressed in the paper Omura and Nunn [2011] for the case of a rising tone. From the numerical results for the faller we see that nonlinear frequency shift $\omega_{1}$ at the generation point during actual triggering climbs to a consistent negative value in a short time designated $T_{N}$, the transition time, though for the case of a faller $\omega_{1}$ undergoes a brief positive excursion first. This time must be of the order of a median trapping time $T_{t r}$ given by

$$
T_{t r}=\frac{2 \pi}{\omega_{t r}}=\frac{2 \pi}{\chi}\left(\frac{m_{0} \gamma}{k V_{\perp o} e B_{w}}\right)^{1 / 2}
$$

where $\omega_{t r}$ is the trapping frequency. We may define a ratio $\tau$ $=T_{N} / T_{t r}$ of order unity and which may be determined from numerical simulation. From (26) we see that if a frequency shift $\omega^{\prime}$ is imposed in a time $T_{N}$ this should result in a temporal frequency sweep rate

$$
\partial \omega / \partial t=\omega_{1} / T_{N}
$$

and the corresponding spatial gradient [Omura and Nunn, 2011].

$$
\partial \omega / \partial h=\omega_{1} /\left(T_{N} V_{g}\right)
$$

We may now find an overall expression for the sweep rate under the assumption of $S=+0.4$ as

$$
\frac{\partial \omega}{\partial t}=\frac{1.3}{4} \pi^{-5 / 2} \frac{Q}{\tau}\left(\frac{\omega_{p h} V_{\perp o} \chi}{\gamma c}\right)^{2} \frac{V_{g}}{U_{t \|}} \exp \left(-\frac{\gamma^{2} V_{R}^{2}}{2 U_{t \|}^{2}}\right)
$$

If we examine the steep onset of $\omega_{1}$ at $\tilde{t}=4.3 \times 10^{4}$ and substitute into equation (28) we obtain a frequency sweep rate $\sim-9 \times 10^{-7}$ in dimensionless units, or $-830 \mathrm{~Hz} / \mathrm{s}$, indeed close to the actual sweep rate $\sim-800 \mathrm{~Hz} / \mathrm{s}$. More interestingly we may substitute simulation parameters into the general theoretical expression equation (30). From Figure 5 we estimate the average distribution function in the trap as being elevated by $\sim+12 \%$ giving $Q=-0.12$. Utilizing parallel thermal energy being $1.5 \mathrm{keV}$ and taking the factor $\tau=0.25$, the theoretical sweep rate is $-837 \mathrm{~Hz} / \mathrm{s}$ in good agreement with the sweep rate of the actual simulation. This value for $\tau$ agrees with the value found in Omura and Nunn [2011] when the theory was applied to a rising frequency emission.

\section{Discussion and Conclusions}

[35] We have noted that broadband PIC simulations and narrow band Vlasov simulations of the triggered emission problem and of VLF chorus either do not trigger falling tones or find it difficult to do so in a stable and repeatable way. In a parabolic inhomogeneity region the negative inhomogeneity region downstream from the equator where risers are triggered will tend to dominate. The difficulty in triggering fallers and their poor quality when triggering is achieved is seen to be due mainly to three factors.

[36] First we note that the emission GR is not a $1 \mathrm{D}$ object but a 3D one, more like a narrow cylindrical 'hot spot' aligned with the ambient field [Santolik et al., 2003]. The GR was shown to be a fairly small structure in the plane perpendicular to Bo. Fourier transformation of the GR field must then give a broad range of $\mathrm{k}$ vector directions about the Bo direction, which will incur substantial Landau damping. Fields generated by the nonlinear resonant particle current field within this GR radiate in $3 \mathrm{D}$ space, probably in a fairly narrow cone of angles $\sim 20$ degrees, since the group velocity distribution will be more tightly confined to the Bo direction than the $\mathrm{k}$ vector distribution. We thus would expect both spreading loss plus Landau damping due to oblique propagation. Hence the faller generated fields are not transmitted loss free to the riser region, but may be considerably damped which would reduce nonlinear trapping in the $\mathrm{S}<0$ zone.

[37] Second it is clear that both PIC and VHS codes assume phase coherence of the wave particle interaction process across the entire simulation region and thus across the equator. In the case of a faller particles will initially become trapped downstream from the equator where wave amplitude is large and $S \sim-1$. In the resonant particle trap we will have a 'hole' $(\mathrm{d} F<0)$, with positive values of integrated energy change $\mathrm{d} W$ and integrated magnetic moment change $\mathrm{d} \mu$, and thus $J_{E}<0$ and $J_{B}<0$. When these trapped particles cross the equator into the $S>0$ zone there will still be a hole in phase space at the trap location, and a 'hill' will only appear as a weaker feature further upstream. Thus the 'memory' of trapping in the $\mathrm{S}<0$ region degrades the trapping process in the $S>0$ region, and results in smaller $J_{B}$, smaller sweep rates and general lack of robustness. Third when the VHS code does trigger a faller the wave profile will often slip downstream into the $S<0$ region creating a riser GR and giving an upward hook.

[38] In order to permit faller production the phase coherence/interaction length for resonant particles needs to be reduced-in particular phase coherence across the equator. A number of factors will act to limit the interaction length. First spectral broadening with respect to both frequency and/or propagation angle will degrade the trapping process and limit interaction length. Second it should be noted that the mean trajectory of wave energy as defined by the group velocity vector and the energetic particle trajectories as defined by their drift motion will diverge thus limiting interaction time. More detailed investigation of wave particle interaction coherence length is an important issue and a subject for future research.

[39] Confining the interaction process to the faller upstream region suppresses cross equatorial phase coherent interaction and has indeed enabled simulation of fallers in a stable and repeatable way and permitted us to examine the faller structure in more detail and to compare it with the predictions of theoretical models [Omura et al., 2009; Omura and Nunn, 2011], where good agreement is obtained for sweep rate.

[40] The faller generation region is now seen to be characterized by positive inhomogeneity factor $S$, by a positive 
current $J_{B}$ parallel to wave magnetic field, a negative nonlinear frequency shift $\omega_{1}$ and by a 'hill' in distribution function in velocity space corresponding to the trap location. All these factors are the exact opposite of those prevailing in a riser GR.

[41] The $-J_{E}$ field highlighted a point at $\tilde{h}=-1300$ $(\sim-5200 \mathrm{~km})$ where $-J_{E}$ was maximal and which could be interpreted as the generation point for the faller emission. The corresponding point for the riser emission GR is located at the equator. Computation of the nonlinear frequency shift $\omega_{1}$ as a function of time at the generation point during the triggering phase revealed a sharp rise to a sustained negative value within a transition time of the order of a trapping time. A similar pattern was found in the case of a riser except that the nonlinear frequency shift was positive. As in the case of the riser results it was found that the sweep rate was roughly the nonlinear frequency shift divided by the transition time and in good accord with theoretical predictions.

[42] An important issue is the one of the relative preference for triggering risers or fallers. It would appear that if trans equatorial propagation is good and the interaction/ coherence length is long then one would expect a strong preference for risers as exhibited by current codes. On the other hand if there is significant propagation loss across the equator and/or interaction/coherence length is short then risers and fallers should compete on more equal terms.

[43] Important experimental results were obtained by the Stanford group with their Siple active experiment. A sequence of pulses were transmitted with increasing length and it was found that shorter pulses triggered fallers which became risers with increasing pulse length.[Helliwell, 1983; Helliwell and Katsufrakis, 1974; Helliwell et al., 1964, 1980].

[44] If we are in a situation where cross equatorial coherence does occur then fallers would tend to be suppressed. However, a short input triggering pulse of reasonable amplitude would naturally prevent cross equatorial interaction and permit a faller GR to be formed. Once a faller GR is established the evidence is that further GR's will not be established at the same time. Some experimental observations show many emissions, both risers and fallers, are triggered by the same pulse, though it is likely in these cases that their GR's are separated in the plane perpendicular to $h$. A longer pulse will allow cross equatorial coherence to suppress the faller allowing the riser only to be produced. We surmise then that the Siple results correspond to a situation in which cross equatorial coherence exists.

[45] In a situation where cross equatorial coherence is small then risers and fallers should compete more equally. In that case other factors need to be considered. The input pulse needs to be linearly amplified to attain trapping amplitude by the time it reaches the GR site in order to trigger. The faller GR location is reached first so one might expect that given a weak triggering pulse or low linear growth rates we will only trigger risers. Yet another aspect is that in short pulses trapping time will be limited and thus the strength of the nonlinear interaction will be weaker at least until a GR is established.

[46] In Nunn et al. [2005] the VHS code was run to trigger emissions and various parameters were systematically changed to see how sweep rate depended on them. One of these parameters was input pulse length. The result was that short pulses did not trigger because trapping time was too short for nonlinearity. With longer pulses the tendency was for risers to be produced. Faller triggering by short pulses was not apparent. One might have expected the VHS code with a full simulation box in $\mathrm{h}$ and embodying cross equatorial coherence to trigger fallers with short input pulses. However, only termination triggering of very weak fallers was noted.

[47] The above arguments are somewhat speculative and this issue needs a lot more research. A suitable tool would be a modified VHS or PIC code in which cross equatorial coherence or interaction time is limited. This is a subject for further research.

[48] An important issue again requiring much further work is the issue of generation region location and its confirmation from satellite observations. In Helliwell's phenomenological theory [Helliwell, 1967], it was supposed that the generation region was an object of small size and located at the point $S=0$, although this point is in fact a function of perpendicular velocity. Recent simulations show that the GR must be of finite size and may occupy a region delimited by the condition $|S|<1$. These simulations seem to emphasize the GR location as being dependent on the sign of $S$ and thus the sign of $J_{B}$, so risers would appear to come from the downstream region and fallers from the upstream region. Paradoxically this is the opposite of Helliwell's $S=0$ criterion. A very comprehensive study [Lauben et al., 2002] using data from POLAR places the generation region near the equator, though assuming oblique propagation. However, their paper considers fallers to be generated on the downstream side of the equator and vice versa for risers. There is a real need to examine in more detail the Cluster data. This does point to the riser GR being downstream from the equator but we need direct evidence from Cluster that the GR for falling chorus is indeed upstream from the equator albeit by only $\sim 2500 \mathrm{~km}$. An obvious item of further work is to run the codes [Hikishima et al., 2010a, 2010b] with the simulation restricted to the upstream region to see if similar results are obtained.

[49] Acknowledgments. D.N. gratefully acknowledges a Visiting Professorship at Kyoto University in 2010. Computations in the present study were performed with the KDK system of the Research Institute for Sustainable Humanosphere and the Academic Centre for Computing and Media Studies at Kyoto University as a collaborative research project. The present study was supported in part by Grant-in-Aid 23340147 and 23224011 of the Ministry of Culture, Sports, Science, and Technology in Japan.

[50] Robert Lysak thanks the reviewers for their assistance in evaluating this paper.

\section{References}

Helliwell, R. A. (1967), A theory of discrete VLF emissions from the magnetosphere, J. Geophys. Res., 72, 4773-4790, doi:10.1029/JZ072i019p04773.

Helliwell, R. A. (1983), Controlled stimulation of VLF emissions from Siple Station, Antarctica, Radio Sci., 18(6), 801-814, doi:10.1029/ RS018i006p00801.

Helliwell, R. A., and J. Katsufrakis (1974), VLF wave injection into the magnetosphere from Siple Station, Antarctica, J. Geophys. Res., 79(16), 2511-2518, doi:10.1029/JA079i016p02511.

Helliwell, R. A., J. Katsufrakis, M. Trimpi, and N. Brice (1964), Artificially stimulated very low frequency radiation from the ionosphere, J. Geophys. Res., 69(11), 2391-2394, doi:10.1029/JZ069i011p02391.

Helliwell, R. A., D. L. Carpenter, and T. R. Miller (1980), Power threshold for growth of coherent VLF signals in the magnetosphere, J. Geophys. Res., 85(A7), 3360-3366, doi:10.1029/JA085iA07p03360. 
Hikishima, M., S. Yagitani, Y. Omura, and I. Nagano (2009), Full particle simulation of whistler-mode rising chorus emissions in the magnetosphere, J. Geophys. Res., 114, A01203, doi:10.1029/2008JA013625.

Hikishima, M., Y. Omura, and D. Summers (2010a), Self-consistent particle simulation of whistler-mode triggered emissions, J. Geophys. Res., 115, A12246, doi:10.1029/2010JA015860.

Hikishima, M., Y. Omura, and D. Summers (2010b), Microburst precipitation of energetic electrons associated with chorus wave generation, Geophys. Res. Lett., 37, L07103, doi:10.1029/2010GL042678.

Katoh, Y., and Y. Omura (2006), A study of generation mechanism of VLF triggered emission by self-consistent particle code, J. Geophys. Res., 111, A12207, doi:10.1029/2006JA011704.

Katoh, Y., and Y. Omura (2007), Computer simulation of chorus wave generation in the Earth's inner magnetosphere, Geophys. Res. Lett., 34, L03102, doi:10.1029/2006GL028594.

Lauben, D. S., U. S. Inan, T. F. Bell, and D. A. Gurnett (2002), Source characteristics of ELF/VLF chorus, J. Geophys. Res., 107(A12), 1429, doi:10.1029/2000JA003019.

Li, W., et al. (2010), THEMIS analysis of observed equatorial electron distributions responsible for the chorus excitation, J. Geophys. Res., 115, A00F11, doi:10.1029/2009JA014845.

Nunn, D. (1974), A self consistent theory of triggered VLF emissions, Planet. Space Sci., 22, 349-378, doi:10.1016/0032-0633(74)90070-1.

Nunn, D. (1986), A nonlinear theory of sideband stability of ducted whistler mode waves, Planet. Space Sci., 34(5), 429-451, doi:10.1016/0032-0633 (86) $90032-2$.

Nunn, D. (1990), The numerical simulation of VLF nonlinear wave particle interactions in collision free plasmas using the Vlasov Hybrid Simulation technique, Comput. Phys. Commun., 60, 1-25, doi:10.1016/0010-4655 (90)90074-B

Nunn, D. (1993), A novel technique for the numerical simulation of hot collision free plasma; Vlasov Hybrid Simulation, J. Comput. Phys., 108(1), 180-196, doi:10.1006/jcph.1993.1173.

Nunn, D., M. J. Rycroft, and V. Y. Trakhtengerts (2005), A parametric study of the numerical simulation of triggered VLF emissions, Ann. Geophys., 23, 3655-3666, doi:10.5194/angeo-23-3655-2005.
Nunn, D., O. Santolik, M. Rycroft, and V. Trakhtengerts (2009), On the numerical modelling of VLF chorus dynamical spectra, Ann. Geophys., 27, 2341-2359, doi:10.5194/angeo-27-2341-2009.

Omura, Y. and H. Matsumoto (1982), Computer simulations of basic processes of coherent whistler wave particle interactions in the magnetosphere, J. Geophys. Res., 87, 4435-4444, doi:10.1029/JA087iA06p04435.

Omura, Y., and H. Matsumoto (1985), Simulation study of frequency variations of VLF triggered emissions in a homogeneous field, J. Geomagn. Geoelectr., 37, 829-837, doi:10.5636/jgg.37.829.

Omura, Y., and D. Nunn (2011), Triggering process of whistler mode chorus emissions in the magnetosphere, J. Geophys. Res., 116, A05205, doi:10.1029/2010JA016280.

Omura, Y., D. Nunn, H. Matsumoto, and M. J. Rycroft (1991), A review of observational, theoretical and numerical studies of VLF triggered emissions, J. Atmos. Terr. Phys., 53, 351-368, doi:10.1016/0021-9169(91) 90031-2

Omura, Y., N. Furuya, and D. Summers (2007), Relativistic turning acceleration of resonant electrons by coherent whistler-mode waves in a dipole magnetic field, J. Geophys. Res., 112, A06236, doi:10.1029/ 2006JA012243.

Omura, Y., Y. Katoh, and D. Summers (2008), Theory and simulation of the generation of whistler-mode chorus, J. Geophys. Res., 113, A04223, doi:10.1029/2007JA012622.

Omura, Y., M. Hikishima, Y. Katoh, D. Summers, and S. Yagitani (2009), Nonlinear mechanisms of lower-band and upper-band VLF chorus emissions in the magnetosphere, J. Geophys. Res., 114, A07217, doi:10.1029/ 2009JA014206.

Santolik, O., and D. A. Gurnett (2003), Transverse dimensions of chorus in the source region, Geophys. Res. Lett., 30(2), 1031, doi:10.1029/ 2002GL016178.

Santolik, O., D. A. Gurnett, and J. S. Pickett (2003), Spatio-temporal structure of storm time chorus, J. Geophys. Res., 108(A7), 1278, doi:10.1029/ 2002JA009791.

Trakhtengerts, V. Y. (1995), Magnetosphere cyclotron maser: BWO generator regime, J. Geophys. Res., 100, 17,205-17,210, doi:10.1029/ 95JA00843.

Trakhtengerts, V. Y. (1999), A generation mechanism for chorus emission, Ann. Geophys., 17, 95-100. 